\title{
Blobs in Wolf-Rayet Winds: Random Photometric and Polarimetric Variability
}

\author{
Cláudia V. Rodrigues ${ }^{1}$ \\ Instituto Nacional de Pesquisas Espaciais/MCT \\ Av. dos Astronautas, 1758 \\ 12227-900 - São José dos Campos - SP \\ Brazil \\ e-mail: claudia@das.inpe.br \\ and \\ A. Mário Magalhães \\ Instituto Astronômico e Geofísico, Univ. de São Paulo \\ Av. Miguel Stéfano, 4200 \\ 04301-904 - São Paulo - SP \\ Brazil \\ e-mail:mario@argus.iagusp.usp.br
}

\begin{abstract}
Some isolated Wolf-Rayet stars present random variability in their optical flux and polarization. We make the assumption that such variability is caused by the presence of regions of enhanced density, i.e. blobs, in their envelopes. In order to find the physical characteristics of such regions we have modeled the stellar emission using a Monte Carlo code to treat the radiative transfer in an inhomogeneous electron scattering envelope. We are able to treat multiple scattering in the regions of enhanced density as well as in the envelope itself. The finite sizes of the source and structures in the wind are also taken into account. Most of the results presented here are based on a parameter study of models with a single blob. The effects due to multiple blobs in the envelope are considered to a more limited extent. Our simulations indicate that the density enhancements must have a large geometric cross section in order to produce the observed photopolarimetric variability. The sizes must be of the order of one stellar radius and the blobs must be located near the base of the envelope. These sizes are the same inferred from the widths of the sub-peaks in optical emission lines of Wolf-Rayet stars. Other early-type stars show random polarimetric fluctuations with characteristics similar to those observed in Wolf-Rayet stars, which may also be interpreted in terms of a clumpy wind. Although the origin of such structures is still unclear, the same mechanism may be working in different types of hot stars envelopes to produce such inhomogeneities.
\end{abstract}

\footnotetext{
${ }^{1}$ formerly at Instituto Astronômico e Geofísico, Univ. de São Paulo
} 
Subject headings: circumstellar matter — methods: numerical — polarization — radiative transfer — stars: early-type — stars: Wolf-Rayet 


\section{Introduction}

Our current understanding of hot star envelopes pictures them as inhomogeneous and non-steady structures (Moffat et al. 1994; Wolf, Stahl \& Fullerton 1999; Magalhães \& Rodrigues 1999). In particular, Wolf-Rayet (WR) stars can present random variations in broad band flux, polarization and also in spectral line profiles. The broad band fluctuations can reach $10 \%$ in flux (Antokhin et al. 1995; Marchenko et al. 1998 ) and $0.5 \%$ in polarization (St.-Louis et al. 1987; Drissen et al. 1987; Robert et al. 1989; Moffat \& Robert 1991). The observed changes in the spectral line profiles can be divided in two types. One of them is the small moving bumps which appear superposed on optical emission lines (Robert 1994). Moffat \& Robert (1992) suggested that they are related to localized enhancements of material, i.e. blobs, in WR winds. The other type of variation seen in WR stars are discrete absorption components (DACs; Prinja \& Smith 1992). In other hot stars, such as OB stars, DACs are also observed and seem to be connected to the rotation period of the star (Kaper \& Henrichs 1994). The DACs in WR stars may then be not related to the random broad band fluctuations, since in other hot stars these features seem to be periodic. Some kind of periodic line variability is seen in a few isolated WR stars - WR 1, WR 6 and WR 134 (see Lepine, Eversberg \& Moffat 1999 and references therein). However, for the stars having polarization measurements (WR 6 and WR 134), the position angle of the polarization is relatively constant, a behavior different from that seen in most of the isolated WR stars which present randomly varying position angles. Therefore the isolated WR stars which present some kind of periodic behavior seem to also have envelopes with a time-independent anisotropy, in contrast to the randomly variable stars. Less direct evidence for clumpy WR winds can be obtained from the fits of line wings (Hamann \& Koesterke 1998; Hillier 1984).

There is evidence that the random variability in the three types of observations (flux, polarization, and spectra) are different facets of the same phenomenon: (1) these variability types are correlated (Robert 1994); (2) the time scales are the same, ranging from hours to days (Moffat \& Robert 1991). Robert (1994) has also shown that the spectral variations are caused by localized density enhancements whose frequency of occurrence increases for smaller masses. The size distribution of these spectral fea- tures is also addressed in Lepine \& Moffat (1996, 1999). The velocity dispersion in these features suggests a physical dimension around one stellar radius for such blobs (Robert 1994).

Theoretical results indicate that hot star envelopes are indeed not expected to be homogeneous. In his review, Owocki (1994) has suggested that early-type star winds can have two types of instabilities. There is a small-scale instability, which is intrinsic to a radiative wind and can be responsible for the bumps observed in the WR emission lines (see also Gayley \& Owocki 1995). On the other hand, external processes, such as rotation, binarity, and photospheric processes, can induce instabilities on large scales. While this second mechanism may produce the DACs, the former may be associated with the so-called blobs in WR winds.

The determination of the physical properties of the inhomogeneities in WR wind constrains the theoretical models for their origin as well as the models for the mechanism producing the wind itself. In the last few years, some studies have tried to model the random polarimetric variations in hot stars (Fox \& Henrichs 1994; Brown 1994; Brown et al. 1995; Code \& Whitney 1995; Richardson, Brown \& Simmons 1996). All these models take into account only the region of enhanced density, consequently the envelope itself is not considered as an element to modify the radiation coming from the star or that scattered by a blob. Most of them are restricted to point-like regions and single scattering treatment, the exception being the numerical Monte Carlo treatment of Code \& Whitney (1995). Studying the random photopolarimetric variability in WR stars, Brown (1994) has concluded that the density enhancements may be originated at the stellar surface and not be the result of a mass redistribution process in the wind, such as turbulence. In a subsequent paper, Brown et al. (1995) have pointed out that the fractional variation in flux and polarization of the continuum light must be of a similar magnitude if they are produced by scattering of starlight, a fact which is not observed; as mentioned early in this paper, the fluctuations in broad band flux are much larger than the polarization. Richardson et al. (1996) have revisited this problem considering an envelope with many blobs and concluded that the observed ratio between the photometric and polarimetric variations, $\Delta I / \Delta P$, cannot be reproduced by any number of inhomogeneities. They have suggested that the emission from blobs might be an important 
contributor to the total flux and this would bring the models closer to the observations. In order for this be valid, the blobs have to be very dense.

In this work, we investigate whether blobs can explain the changes in flux and polarization observed in WR stars and what would their resultant physical characteristics be, as the size and distance from the star. We have included, besides scattering, extinction as a mechanism to produce flux variations. We have used a Monte Carlo code that does not have the limitations of single scattering or point-like sources. Unlike earlier works, scattering in the envelope itself is also considered. This is important since WR stars have optically thick envelopes. In the following section, we introduce the main features of our model. In Section 3, we describe how the resulting broad band variations depend on the input parameters for a model with a single blob. In this section, some results for envelopes with more than one blob are also presented. The model results are applied to WR envelopes in Section 4. The applicability of the model for other hot stars and a summary are then presented.

\section{The model}

\subsection{Model assumptions}

We assume that the observed broad band random variability in WR stars is caused by the presence of regions of enhanced density which we will call blobs. Here we are not interested in explaining their origin: our goal is just to constrain the physical characteristics that the blobs may have in order to explain the observed quantities. We have solved the radiative transfer in an electron scattering envelope using the Monte Carlo code described in Rodrigues (1997). This code has already been used to study the light curve and polarization of WR binary stars (Rodrigues \& Magalhães 1994; Rodrigues 1997). The basics of a Monte Carlo code to simulate radiative transfer is described in Code \& Whitney (1995).

The blobs have been assumed to be spherical and immersed in a spherical envelope. The density law of the envelope can be chosen among many analytical expressions: constant, inversely proportional to the square of the distance, or following a beta-type law for the wind velocity in radiative driven winds $\left.\left[v(r) \propto\left(1-R_{\star} / r\right)^{\beta}\right)\right]$, among others. The density within the blob follows the same law as the envelope, but it is multiplied by an arbitrary factor which intro- duces a density enhancement. For instance, if this factor is equal to one, the envelope is isotropic (no blobs exist at all). Therefore, our model does not represent density enhancements caused by redistribution of matter in the envelope. It is consistent with Brown's (1994) statement that polarization is not caused by a process with such a characteristic. In addition, we are able to treat an arbitrary number of blobs $\left(N_{b l}\right)$. The only source of radiation is an extended, spherical central source, i.e., the envelope and the blobs do not emit any radiation. The use of the Monte Carlo method has allowed us to consider optically thick envelopes characteristic of WR winds. The last point is an important difference in comparison with previous models, which have considered no envelope at all.

The number of photons $(N)$ of each simulation was either $3 \times 10^{7}$ or $3 \times 10^{8}$ for optically thin and thick envelopes, respectively. The exiting photons were classified among 400 bins of identical solid angle. The light emitted by the source was considered to be isotropic and not polarized. Some of the photons can hit the source. Initially, we have considered they were absorbed by the source. However, reemission of these photons may in principle have an effect on the results 2. We have then modified our code in order to include the backwarming of the stellar photosphere. This was done by isotropically reemitting the photon at the point it hits the star. We found that the fractional differences of the intensity and polarization values $(\Delta I$ and $\Delta P$; see end of Section 2.2 for their definition) which characterize a given model are less than 0.0044 and 0.023 respectively. These differences are small and within the errors of the simulations (see Section 2.2). All the results presented in this work, consider the backwarming.

A spherically symmetric envelope produces a given total amount of non-polarized flux independent of the observer's direction. In contrast, the flux and polarization of an envelope with one or more blobs depend on the direction of the observer. Consequently, the photopolarimetric variability may arise in two situations: (1) when the wind changes from a homogeneous configuration to an inhomogeneous one; or (2) when the wind is always inhomogeneous, but with blobs whose position relative to the observer is variable. The resultant variations from situation (2) may actually be observationally very similar to case (1).

\footnotetext{
${ }^{2}$ We wish to thank J. Bjorkman for pointing that out and discussing it with us.
} 
This is because an envelope with a blob may be practically indistinguishable from a homogeneous, one except if observed under some lines of sight (see Section 2.2 and Figure 11). A moving blob may then cause a change in the observed flux and polarization.

The input parameters of the models are listed in Table 11 as well as the range of values for which we have obtained results. There is a relatively large number of input parameters but, as we will see in Section 4.1, we were able to constrain some of them based on the observed variation of the intensity and polarization. The results do not depend on the linear scale of the model. If we modify the source, blob and envelope sizes using a same arbitrary multiplicative factor, the output is exactly the same, provided the optical depth of the envelope is kept the same. Thus all the parameters with units of length have been normalized to the radius of the source. Because of that, the source radius is not strictly an input parameter. All the models have used the same source and external envelope radii (Table 1). The density of most models has been considered as constant. We have indicated where we have used another density law.

The reliability of the results has been tested in different ways. For instance, our code provides values of the polarization consistent with zero in isotropic configurations and the results do not change if the scale of the problem varies. Other quantitative tests were performed by comparing our results with those of previous calculations. We have used for the latter: (1) the analytical solution of Chandrasekhar (1950) for a semi-infinite plane-parallel optically thick envelope; (2) the Monte Carlo solution of Lefevre \& Daniel (1988) for a dusty envelope; (3) the Monte Carlo solution of Code \& Whitney (1995) for a blob illuminated by a plane-parallel beam; (4) the analytical results of Brown (1994). Our results are consistent with all the above calculations. Graphs and tables can be found in Rodrigues (1997).

\subsection{Model output}

The code provides values of the flux, linear polarization and its position angle, and the circular polarization as a function of the line of sight under which the system is observed. These directions are represented by a spherical coordinate system centered on the star: $\theta$ represents the polar angle and $\phi$, the azimuthal angle. The photons are classified in bins of same solid angle whose centers are equally spaced in $\phi$ and $\cos \theta$ (it is necessary to use $\cos \theta$ for the bins to have the same solid angle). The emerging circular polarization is null in an electron scattering wind, if no circular polarization is input at the base of the wind, since this process does not introduce any phase shift upon the scattered wave. The flux is normalized to the value of a homogeneous wind. Here, a value equal to one means that the flux is the same as that observed from a wind without blobs.

Figure 11 shows an example of the angular dependence of the flux and polarization obtained with our code. The blob is located at $\theta=90^{\circ}$ and $\phi=180^{\circ}$. The input parameters of this model are given in the caption. The flux as a function of the line of sight direction, shown in Figure 1 a, has a relatively simple pattern. The flux has been normalized to that of a homogeneous envelope, $I_{o}$, so $I / I_{o}$ is shown. The central dip is caused by extinction by the blob. The light extinguished from along the direction of the blob is scattered to all directions according to the phase function of the scattering process which causes a flux modulation away from the line of sight to the blob. In the figure, the maximum of the scattered flux around $90^{\circ}$ from the blob direction can be noticed. This increase in flux is much smaller than the decrease caused by extinction along the blob direction.

Figure 1 b illustrates the polarization pattern which is primarily determined by the electron scattering phase function. Therefore, the polarization is approximately zero near the direction of the blob and near the opposite direction $\left(\theta=90^{\circ}, \phi=0^{\circ}\right)$. Its maximum value is reached near $90^{\circ}$ from the blob direction; the actual angle depends on the optical depths of the envelope and blob.

The errors in our Monte Carlo simulations follow a Poisson statistics; consequently they decrease as $\sqrt{N}$ when a larger number of photons is used. For the number of photons and solid angle bins used, the uncertainty in the normalized intensity values derived using the Poisson statistics is in the third significative digit: $3.6 \times 10^{-3}$ for $3 \times 10^{7}$ photons and $1.2 \times 10^{-3}$ for $3 \times 10^{8}$ photons. These values agree with the standard deviation of the intensity in regions where it is expected to be constant.

In order to estimate the error in the polarization, we have run some models for which we expect a null value of the polarization for any direction. In this case, the average value of the polarization can be assumed to give an estimate of the uncertainty. This value is practically the same as the Poisson error derived from all the simulated photons: $0.018 \%$ for 
$3 \times 10^{7}$ photons and $0.0057 \%$ for $3 \times 10^{8}$ photons .

An additional remark should be made about the polarization errors. Because the polarization is an absolutely positive number, its error biases the polarization towards larger values: the best estimate to the polarization is given by $\sqrt{P_{o b s}^{2}-\sigma_{P}^{2}}$, where $P_{o b s}$ is the observed polarization and $\sigma_{P}$ is the associated error (Clarke \& Stewart 1986). Because of that, the polarization degrees obtained in the simulations may overestimate the actual values.

In order to simplify the analysis, each model was reduced to two values: the maximum variation of the flux, $\Delta I$; and an estimate of the maximum polarization, $\Delta P$, as described below. These values are the ones to be compared with the WR photometric and polarimetric variations. The value of $\Delta I$ is given by: $1-\left(I_{\min } / I_{o}\right)$, where $I_{\min }$ is the minimum value of the flux. The value of $\Delta I$ for the model in Figure 17a is $67 \%$. In doing that, we assume that the flux variation in WR stars is produced by the passage of a blob in the line of sight causing a decrease in the total flux from the star, i.e. the changes in flux are due to the extinction. As illustrated by Figure 1, the blobs also scatter, so the flux increases along directions which do not intercept the blob. However, as we will further discuss towards the end of Section 3.1 and in Section 3.2, this variation is smaller than that caused by extinction if $N_{b l}$ is less or around 20, for optically thin envelopes.

The polarization from a homogeneous envelope is zero in any direction. The polarization caused by an envelope with blobs can be then considered as the polarimetric variation itself. The maximum polarization was estimated in the following way. We first calculated the average polarization for bins of same $\theta$. The value of $\Delta P$ was chosen as the maximum one from such averages. This procedure enables us to have also an estimate of the variance of the maximum polarization. It also avoids any bias that may result from selecting the largest absolute value among all the bins. It could be argued that the bins of same $\cos \theta$ do not all form the same angle with the blob, so that an average might not be appropriate. However, given that the bin sizes are relatively large and that the maximum polarization occurs at angles close to $90^{\circ}$ from the blob, this average is a valid approximation. The value of $\Delta P$ so calculated for the model shown in Figure 1 $1 \mathrm{~b}$ is $0.86 \% \pm 0.08 \%$. The maximum value of the polarization for the same model is $0.98 \%$.

\section{General results}

In this section, we present some numerical results which are helpful in order to understand the model physics. We first present the dependence of the results on the input parameters. We then show specific results contrasting the extinction and the scattered flux obtained with the model. We do not contend that all models here presented are valid representations of inhomogeneous WR envelopes, since some of them are very optically thin.

\subsection{Dependence of the results on the input parameters}

A general drawback of Monte Carlo simulations of physical problems is that the influence of a given parameter may tend to be lost in the final result. To gain an insight into this influence, we present in this section the behavior of $\Delta I$ and $\Delta P$ as function of some parameters of the model.

Optical depth of the envelope The dependence of $\Delta I$ and $\Delta P$ with the optical depth of the envelope is shown in Figure 2 (solid and dotted lines). The polarimetric and photometric variations decrease as $\tau_{\text {env }}$ increases. This happens because the higher the envelope density, the higher the number of scatterings in the envelope which tends to mask any effect produced by the blob.

Optical depth of the blob Figure 2 also illustrates the effect of increasing the optical depth of the blob. For very small values, when single scattering dominates, $\Delta I$ and $\Delta P$ increase linearly with $\tau_{b l}$ (it cannot be properly seen in the figure because of the abscissa scale). With further increase in the blob optical depth, multiple scattering becomes more important and $\Delta I$ and $\Delta P$ increase much more slowly and do not seem to exceed a limiting value. The radiation can penetrate optically thin blobs, so all the scatterers contribute to the variations, specially the polarimetric ones. On the other hand, for optically thick blobs, only the scatterers placed in the region facing the source play a role in the polarized flux. Both $\Delta I$ and $\Delta P$ then level off with the increase in $\tau_{b l} ; \Delta P$ levels off at a smaller value of $\tau_{b l}$ compared to $\Delta I$ since the polarization is more sensitive to the increase in optical depth. 
Distance of the blob to the central source The solid angle subtended by the blob decreases when it is located farther from the source. Therefore, a smaller fraction of the source radiation is intercepted by the blob and $\Delta P$ decreases (Figure 2; compare the solid and dashed curves). The average optical depth of a bin, particularly that of the bin including the direction passing through the blob center, is smaller if the blob is located at a larger distance from the source, so $\Delta I$ also decreases with distance (Figure 2). Note that the effect on $\Delta P$ is real and does not depend on the bin size, while that on $\Delta I$ is caused by the finite spatial resolution of the models.

Blob size Figure 3 shows the behavior of $\Delta I$ and $\Delta P$ as a function of blob size. It can be noticed that the variations depend strongly on the blob dimension. This rapid increase of $\Delta I$ and $\Delta P$ with $R_{b l}$ is connected with two important physical parameters: the solid angle of the blob and the total number of scatterers in the blob. Each of these parameters dominates over a certain range of the blob optical depth. In the optically thin limit, all particles in the blob effectively scatter the light. However, for optically thick blobs, only the particles near the source are important in the scattering process. In larger blobs the area facing the source is larger, so larger is the overall variation in intensity and polarization of the emerging flux. Comparing two models of same blob optical depth, $\tau_{b l}$, but different sizes, $R_{b l}$, we note that the blob having a larger size has a smaller density in order to keep the same optical depth.

Different density laws In Figure 1 , we compare the results for two different density laws: uniform and beta laws. We have adopted a beta coefficient of 0.5 in these runs. This is the value obtained for the most simple calculations of a radiative wind (Castor, Abbott \& Klein 1975). We recall that the blob follows the same density law as the envelope. The dependence of $\Delta I$ and $\Delta P$ with $\tau_{b l}$ are shown for the optically thin $\left(\tau_{e n v}=0.01\right)$ and thick $\left(\tau_{e n v}=1\right)$ cases. In the latter, in spite of the differences in the distribution of matter along the envelope, the variations in the flux and polarization of the emerging light are not considerably affected by the density radial distribution. For the optically thin cases, the differences are larger, particularly in the intensity curves. The optical depth of the model envelope is very small $\left(\tau_{e n v}=0.01\right)$ and the difference cannot be due to scattering in the envelope.
When the matter follows a beta law it is more concentrated near the base. For radial optical paths passing through the center of the blob, the optical depth is the same for the two density laws - this is imposed by the choice of parameters. Other radial paths, however, have smaller optical depth in the case of the beta law. As our solid angle bins have a finite size, they include a range of directions and the effective optical depth in the bin is smaller in the beta law case. This causes the smaller $\Delta I$ for the beta law shown in Figure 4 .

The differences in $\Delta I$ and $\Delta P$ between the two density laws are small considering our relatively simple model and that we do not have very precise observational values to be compared with. For these reasons we consider that uniform envelopes give a sufficient representation for scattering calculations in the present context. This result is important because the beta law calculations are computationally (much) more costly than uniform density ones.

Number of blobs The presence of a number of sub-peaks in emission lines is usually taken as evidence for the presence of more than one blob in WR winds (Robert 1994). The average number of such sub-peaks is around 10 (Robert 1994). It is thus important to understand how $\Delta I$ and $\Delta P$ can be affected by the number of blobs as compared to the values produced by one condensation. This section does not intend to fully explore the possibilities of an optically thick envelope with many blobs; this merits a separate paper and will be done elsewhere. Single scattering calculations for a cloud of point-like blobs have been presented by Richardson et al. (1996).

We have run some models with a number of blobs, $N_{b l}$, between 1 and 75, assuming no superposition among blobs. In Table 2 we summarize the results. All the parameters where considered fixed (see Table2 for their values) across simulations, except for $N_{b l}$ and the blob positions. The exact meaning of the columns is explained in the following paragraphs. Generally speaking, they are averages of a number of simulations (between 3 and 10, see Table 2) with different spatial blob distribution. We have assumed that the blobs have a uniform angular distribution, but with a fixed distance to the source $\left(d_{b l}=3\right)$. The $\sigma$ values are the standard deviations of the sample.

Table 2 shows the average of $\Delta I$ for sets of simulations with the same $N_{b l}$. An increase of $\Delta I$ with 
the number of blobs is apparent. As the spatial resolution of the models is modest, the position of the blob relative to the bin center could have some effect on $\Delta I$. In order to ascertain such effect, we ran 10 simulations with a single blob; in each of them the blob was located at different positions in the envelope. The first line in Table 2 is the average of these simulations. The resultant small error means that $\Delta I$ is quite independent of the blob position. Thus the increase in $\Delta I$ with $N_{b l}$ is not caused by effects related to the blob position, but it seems to be a consequence of the presence of more than one blob in the envelope. The explanation for this effect is the following. Let's consider only photons emitted by the source in one direction. They form a beam whose cross section has the size of the stellar disk $\left(\pi R_{s}^{2}\right)$. In the simulations we have run, the cross section of one blob is 0.25 of this value. If the envelope has only one blob, obviously the beam can only cross one blob. But with the increase of $N_{b l}$, more than one blob can be crossed by the same beam: it will depend on the angular distribution of the condensations in each simulation. This explains the increase of $\Delta I$ with the number of blobs and also the larger standard deviation compared with the case of one blob. Table 2 also shows that $\Delta I$ increases until $N_{b l}$ around 30 and then levels off or decreases. This happens because the scattered photons becomes an important fraction of photons leaving the envelope and, as their angular distribution tends to be isotropic, this "emission" tends to fill the dips in the intensity caused by extinction. This effect has the same nature of the decrease of $\Delta I$ when $\tau_{e n v}$ increases (see Figure 2).

In Table 2 we also list the polarization as a function of $N_{b l}$. The fourth column shows the averages of the polarization degree, $\bar{P}$, from a given set of simulations. The fifth column presents the standard deviation of the sample, $\sigma_{P}$; this gives an idea of how wide the distribution of polarization actually is. Figure 5 shows the histograms of the polarization degree for simulations of same $N_{b l}$. The values in Table 2 are then a summary of these histograms. The maximum of $\bar{P}$ occurs around 20 blobs and after that $\bar{P}$ decreases slowly. Around this number, the standard deviation of the polarization also peaks. This may be interpreted as the situation where the anisotropy of the envelope is maximum; this is valid for the configuration represented by the model parameters: blob size, optical depths of blob and envelope, blob distance to the source, and so on. These results may be contrasted with those of Richardson et al. (1996): for distinct model assumptions, the average polarization and variance were always increasing functions of the blob number. The largest number of blobs they have considered was 100 .

\subsection{Extinction versus the scattered flux}

In Table 3, we show some results related to the scattered flux of our models. This table also presents the variation caused by extinction to comparison. In the first three lines, we show the results for one-blob models whose the variation in flux due to scattering, $F_{\text {scat }}$, was the highest for a given optical depth of the envelope. $F_{\text {scat }}$ was calculated considering the largest flux among all the bins in one given simulation. In the remaining lines of this table, we fixed all the parameters of the models, except $N_{b l}$. The values of $F_{\text {scat }}$ are the averages of different simulations with the same $N_{b l}$.

If we consider only the results for one-blob envelopes, we notice that: (1) $F_{\text {scat }}$ is less than $5 \%$ for the optically thicker envelopes; (2) the variation caused by extinction is always greater than that caused by scattering. On the other hand, for envelopes with a large number of blobs $\left(N_{b l}>20\right)$, the amount of scattered flux can be very similar to the extinguished one. The value of $N_{b l}$ for which $F_{\text {scat }}$ becomes important is dependent on the blob parameters (size and optical depth), since it is a main function of the total number of scatterers. Our results for many blobs were obtained for optically thin envelopes. The one-blob results (Table 3) seem to suggest that $F_{\text {scat }}$ will be less important for optically thick envelopes in the many-blob situation as well. This is quite plausible since in optically thick envelopes the flux tends to be more isotropic.

\section{Random broad band variability in WR stars}

\subsection{Simulation results}

Single WR stars may present polarimetric and flux variations of $0.5 \%$ and $10 \%$, respectively. From the previous sections, we can state that the variations observed in flux can be produced by extinction, if $N_{b l}$ is small, or by extinction and scattering, if $N_{b l}$ is larger than around 10, for the parameters used. If the brightness changes are caused by extinction, the polarization will never be observed simultaneously with the variation in intensity produced by the same blob. 
If, for instance, a given blob is along the line of sight towards the center of the star it will cause a decrease in flux, but the (maximum) polarization caused by the same blob will only be noted by an observer in the direction perpendicular to that one. In other words, the largest variations in flux and polarization do not occur along the same line of sight. So it would not in principle be necessary to consider only one type of blob to explain the observations. We nevertheless may assume here without loss of generality that the observed fluctuations are produced by blobs having the same physical characteristics. As we now show, this assumption explains the observed photopolarimetric fluctuations.

Some results for one-blob models with $\tau_{\text {env }}$ greater or equal to 0.1 are shown in Table 4 . They were selected from hundreds of simulations whose parameters are within the ranges presented in Table 1. We have selected models with $\Delta I$ around $10 \%$ and $\Delta P$ greater than $0.2 \%$. Some models have optically thin envelopes which may not correctly represent a WR envelope. However we have kept them to allow a comparison with the optically thick model results. In Table 4 we also include the mass of one blob, $M_{b l}$, as a fraction of the total envelope mass for each model. These values rely on the composition and the degree of ionization not varying strongly throughout the envelope since they depend on the electron density.

A general result is that an extinction value of $10 \%$ is easily achieved for practically any choice of blob and envelope parameters. One needs only to adjust the optical depth of the blob, $\tau_{b l}$. Although it is not shown in Table (because of the associated small values of polarization), models with blobs far from the base of the envelope can also produce $10 \%$ of variation in flux. We conclude that the photometric variation by itself does not strongly constrain the physical characteristics of the blobs.

On the other hand, a polarization of $\approx 0.5 \%$ is only obtained for a few specific cases. In general, a blob produces smaller values of polarization. The highest values of polarization are achieved for an envelope with a large blob near the base of the envelope: for instance, the model with $\tau_{e n v}=1.0, d_{b l}=3, R_{b l}=1.0$ and a beta law for the density is one such case. We conclude that the blobs must have a large geometric cross section in order to produce the observed values of polarization (see also Section 4.2).

We can now address the applicability of these results to the flux variations of WR stars. The simplest scenario is that of an envelope with a single blob. In this situation, we have already shown that the scattered flux does not produce a variation as high as $10 \%$, while the extinction can reach this value easily. The question is, how probable is it to observe such extinction? The exact answer depends on the size, distance to the source, and density distribution of the blob, but we can notice in Figure 1 that the range of directions where we can observe the extinction is considerably large. So the probability of obtaining around $10 \%$ from extinction from the blob is non negligible. With an increase in the number of blobs, this probability increases because there will be more lines of sight which intercept a blob, whereas the angular shape and level of the decrease caused by extinction remains practically the same: this is valid if the blobs are not very close angularly. However, the scattered flux also increases with the number of blobs because each blob contributes individually to the total (see Table 3). We note however that the results for many blobs (end of Section 3.1 and Section 3.2) were obtained for optically thin envelopes: this may overestimate $F_{\text {scat }}$. Therefore, in a more realistic scenario where many blobs coexist, the flux variations will originate from the interplay between two situations: (1) the line of sight intercepts any blob, in which case extinction effects dominate; (2) there is no blob in the line of sight, in which case there is a scattered light contribution dependent of the number of blobs and their spatial distribution. This result contrasts with previous studies (Brown et al. 1995; Richardson et al. 1996), where it was assumed that the flux variation is produced mainly by scattering.

The polarization produced by an inhomogeneous envelope depends in a more complicated way on the envelope parameters than the flux. The main reasons are: (1) polarizations from individual blobs do not sum algebraically; (2) a blob produces polarization in regions not confined to a line of sight. If we consider only one blob, we have shown that a polarization of $0.5 \%$ is only achieved with blobs near (less than about 3 stellar radius) the base of the envelope and whose sizes are similar to the stellar radius. Figure 5 shows some examples of the number distribution of polarization degree for simulations with a varying number of blobs for optically thin envelopes. Diagrams like those can be compared with observational data in order to constrain the physical characteristics of the blob. They would also be helpful in predicting the polarization from a given theoretical blob model. 
This will be explored in a forthcoming paper.

We have not included the intrinsic emission from the envelope and blobs. It would probably tend to smooth the variations in flux and polarization with aspect, since the emission is isotropic and non-polarized.

As stressed by Brown et al. (1995), simultaneous observations in the three mode of observations would be very important for further constraining the blob properties. We are carrying an observational program to observe highly variable WR stars using photopolarimetric and spectroscopic techniques.

\subsection{Analytical calculations for the blob cross section}

A simple argument in favor of a large cross section for a blob in order to cause the observed values of $\Delta I$ and $\Delta P$ can be presented. Let us assume the situation of a blob immersed in a transparent envelope, with the blob and the star in the plane of sky so the scattered flux from the blob has a $90^{\circ}$ scattering angle. The unscattered brightness (light coming directly from the source) received by the observer will be called $I_{d i r}$. We assumed that this component is completely unpolarized.

Assuming that the blob scatters the light it intercepts according to the phase function of the electron scattering, the scattered intensity from a blob to the observer direction, $I_{\text {scat }}$, is:

$$
I_{s c a t}=\frac{3}{8} \frac{1}{2 \pi} I_{d i r} \sigma_{b l},
$$

where $\sigma_{b l}$ is the solid angle of the blob (as seen by the central source) and the numerical factor before $I_{d i r}$ is the probability of scattering in the observer direction. The scattered light is $100 \%$ polarized, so the net polarization is just the ratio between $I_{\text {scat }}$ and $\left(I_{\text {scat }}+I_{d i r}\right)$ (Code \& Whitney 1995).

In this simple scenario, a blob of one stellar radius situated at 2 stellar radius produces a polarization of $4 \%$. If the blob has half a stellar radius, the polarization will be $1 \%$. These values are an overestimate for the polarization: if the blob's optical depth is small, only a fraction of the source light will be scattered; consequently $I_{\text {scat }}$ will decrease and so will the polarization. On the other extreme, if the blob is optically thick, the scattered light, $I_{\text {scat }}$, would be much more isotropic and not $100 \%$ polarized because of multiple scattering inside the blob. In any case, in order to achieve a degree of polarization around $0.5 \%$ the blobs must have large cross sections.

\section{Photopolarimetric variability in other early- type stars}

Taylor et al. (1991) have shown that P Cygni, a luminous blue variable, presents random variations in its optical polarization. Their data, collected during 1989 and 1990, show amplitude variations between $0.04 \%$ and $0.48 \%$, with no preferred value for the position angle. They have pointed out that such a large variation indicates that the material causing polarization must be close to the star and suggested that a clumpy and variable wind must be causing the variation in continuous polarization. Coronographic images of this star shows indeed unresolved clumps of emission distributed asymmetrically in the envelope (Nota et al. 1995). Consequently, the model of an envelope with condensations is consistent with $\mathrm{P}$ Cygni observations.

Polarimetric observations of $\mathrm{OB}$ supergiants have shown that a large fraction of these stars ( 7 out of 10) presents random fluctuation between $0.2 \%$ and 0.4\% (Lupie \& Nordsieck 1987; Bjorkman 1994). The existence of blobs in the wind which scatter the source light has been considered as a possible explanation to this behavior.

It is clear therefore that random variability is also present in other early-type stars besides WR stars. An envelope with inhomogeneities is a possible interpretation in these cases and seems indeed to be the best explanation, at least for the supergiant $\mathrm{P}$ Cyg. Based on the observed polarization variations, the blobs seem to have properties similar to those which explain the WR variability: they must be large and close to the star. This may be an indication that the same phenomenon is working in hot stars with quite distinct winds and causing a similar clumpy structure.

\section{Conclusions}

We have for the first time treated the problem of the multiple scattering in an electron scattering envelope where regions of enhanced density exist. The model results, obtained using a Monte Carlo code, were used to study the random variation in broad band flux and polarization of WR stars. We show that the flux variation amplitude may be due to the extinction caused by blobs intercepting the line of sight, if $N_{b l}$ is smaller than 10. For an envelope with a larger number of blobs, both scattering and extinc- 
tion, may play a role in the flux variations. We show further that the flux variations do not constrain much the physical properties of the inhomogeneities. The polarization variation, on the other hand, used simultaneously with the photometric variation, is a more useful constraint to the blob characteristics. The photopolarimetric variability in WR stars indicates that the inhomogeneities must have relatively large sizes (comparable to that of the star) and be near the base of the photosphere. The envelope may possibly, but not necessarily, have few blobs. If a clumpy structure is causing the polarimetric fluctuations seen in other types of hot stars, the blobs probably have similar characteristics since the amplitude variations are of the same order.

We thank J. Bjorkman for his careful reading of the paper and his valuable comments. We also thank the anonymous referee for his insightful comments and remarks which helped to improved the paper. We are also grateful to Fapesp for the financial support to the polarimetric group at IAG/USP (grants 92/3345-0, 94/0033-3 and 97/11299-2). CVR also thanks Fapesp by its financial support through grants 1992/1812-0 and 1998/1443-1. AMM additionally acknowledges Fapesp grant 98/4267-0 and CNPq grant 301558/795. This research has made use of NASA's Astrophysics Data System Abstract Service. 


\section{REFERENCES}

Antokhin, I., Bertrand, J. F., Lamontagne, R., Moffat, A. F. J., \& Matthews, J. 1995, AJ, 109, 817

Bjorkman, K. S. 1994, Ap\&SS, 221,335

Brown, J. C. 1994, Ap\&SS, 221, 357

Brown, J. C., Richardson, L. L., Antokhin, I., Robert, C., Moffat, A. F. J., \& St-Louis, N. 1995, A\&A, 295,725

Chandrasekhar, S. 1950, Radiative Transfer (Oxford: University Press)

Castor, J. I., Abbott, D. C. \& Klein, R. I. 1975, ApJ, 195,157

Clarke, D., \& Stewart, B. G. 1986, Vistas in Astronomy, 29, 27

Code, A. D., \& Whitney, B. A. 1995, ApJ, 441, 400

Drissen, L., St.-Louis, N., Moffat, A. F. J., \& Bastien, P. 1987, ApJ, 322, 888

Fox, G. K., \& Henrichs, H. F. 1994, MNRAS, 266, 945

Gayley, K. G., \& Owocki, S. P. 1995, ApJ, 446, 801

Hamann, W.-R., \& Koesterke, L. 1998, A\&A, 335, 1003

Hillier, D. J. 1984, ApJ, 280, 744

Kaper, L., \& Henrichs, H. F. 1994, Ap\&SS, 221, 115

Lefevre, J., \& Daniel, J. Y. 1988, in Polarized Radiation of Circunstellar Origin, ed. G. V. Coyne, S. J. et al. (Vatican City State: Vatican Observatory), 523

Lepine, S. \& Moffat, A. F. J. 1996, ApJ, 466, 392

Lepine, S. \& Moffat, A. F. J. 1999, ApJ, 514, 909

Lepine, S., Eversberg, T. \& Moffat, A. F. J. 1999, AJ, 117,1441

Lupie, O. L., \& Nordsieck, K. H. 1987, AJ, 92, 214

Magalhães, A. M., \& Rodrigues, C. V. 1999, in IAU Coll. 169: Variable and Non-spherical Stellar Winds in Luminous Hot Stars, ed. B. Wolf, O. Stahl \& A. W. Fullerton. (Heidelberg: Springer), 49
Marchenko, S. V., Moffat, A. F. J., Eversberg, T., Hill, G. M., Tovmassian, G. H., Morel, T., \& Seggewiss, W. 1998, MNRAS, 294, 642

Moffat, A. F. J., \& Robert, C. 1991, in IAU Symp. 143: Wolf-Rayet Stars and Interrelations with Massive Stars, ed. K. A. van der Hucht \& B. Hidayat (Dordrecht: Kluwer), 109

Moffat, A. F. J., \& Robert, C. 1992, in ASP Conf. Ser. 22: Nonisotropic and Variable Outflows from Stars, ed. L. Drissen, C. Leitherer \& A. Nota (San Francisco: Astron. Soc. of Pacific), 203

Moffat, A. F. J., Owocki, S. P., Fullerton, A. W., \& St.-Louis, N. (ed.) 1994, Instability and Variability of Hot-star Winds, Ap\&SS, 221

Nota, A., Livio, M., Clampin, M., \& Schulte-Ladbeck, R. 1995, ApJ, 448, 788

Owocki, S. P. 1994, Ap\&SS, 221, 3

Prinja, R. K., \& Smith, L. J. 1992, A\&A, 266, 377

Richardson, L. L., Brown, J. C., \& Simmons, J. F. L. 1996, A\&A, 306, 519

Robert, C. 1994, Ap\&SS, 221, 137

Robert, C., Moffat, A. F. J., Bastien, P., Drissen, L., \& St.-Louis, N. 1989, ApJ, 347, 1034

Rodrigues, C. V. 1997, PhD Thesis, Inst. Astronômico e Geofísico, Univ. de São Paulo

Rodrigues, C. V., \& Magalhães, A. M. 1994, in IAU Symp. 163: Wolf-Rayet Stars: Binaries, Colliding Winds and Evolution, ed. K. A. van der Hucht \& P. M. Williams (Dordrecht: Kluwer), 260

St.-Louis, N., Drissen, L., Moffat, A. F. J., Bastien, P., \& Tapia, S. 1987, ApJ, 322, 870

Taylor, M., Nordsieck, K. H., Schulte-Ladbeck, R. E., \& Bjorkman, K. S. 1991, AJ, 102, 1197

Wolf, B., Stahl, O. \& Fullerton, A. W. (ed.) 1999, IAU Coll. 169: Variable and Non-spherical Stellar Winds in Luminous Hot Stars (Heidelberg: Springer), in press

This 2-column preprint was prepared with the AAS LATEX macros $\mathrm{v} 4.0$. 
Fig. 1.- Example of the angular dependence of intensity and polarization obtained as result of a Monte Carlo simulation for the radiative transfer in an inhomogeneous envelope with a single blob of radius $R_{b l}$, located at $\theta=90^{\circ}$ and $\phi=180^{\circ}$ : (a) intensity relative to that of the homogeneous case; (b) percent polarization. The input parameters are: $\tau_{\text {env }}=0.01$; $d_{b l}=3 ; R_{b l}=1 ; \tau_{b l}=10$; uniform density law. The envelope radius, $R_{e n v}$, has been taken as 10 in this and all other simulations in this paper. $R_{e n v}, R_{b l}, d_{b l}$ are normalized to the source radius.

Fig. 2.- Variations of intensity and polarization in one-blob envelopes of different optical depths and at different distances from the source. The points represented by triangles are upper limits to the polarization. The model parameters are: $R_{b l}=1$; uniform density law.

Fig. 3.- Variations of intensity and polarization caused by one blob of variable size. The envelope density follows an uniform law.

Fig. 4.- Variations of intensity and polarization caused by models with different density laws with a single blob. The optical depths of the envelope are 0.01 and 1 for the optically thin and thick cases, respectively. The model parameters are: $d_{b l}=3$; $R_{b l}=1.0$. The beta law coefficient used was 0.5 .

Fig. 5.- Histograms of the number frequency of the polarization degree for simulations with varying $N_{b l}$ (1 through 75). The models parameters are: $\tau_{e n v}=0.01 ; \tau_{b l}=5.0 ; R_{b l}=0.5 ; d_{b l}=3.0$. 
TABLE 1

INPUT PARAMETERS USED TO GENERATE MODELS OF INHOMOGENEOUS WR WINDS

\begin{tabular}{ll}
\hline \hline \multicolumn{1}{c}{ Input Parameters } & \multicolumn{1}{c}{ Range of Values } \\
\hline Source radius, $R_{s}$ & 1.0 \\
Envelope radius, $R_{e n v}{ }^{\text {a }}$ & 10 \\
Envelope optical depth, $\tau_{e n v}$ & $0.01-2$ \\
Blob radius, $R_{b l}{ }^{\text {a }}$ & $0.25,0.5,1.0$ \\
Blob optical depth, $\tau_{b l}$ & $0.1,0.2,0.5,1.0,2.0,5.0,10.0$ \\
Blob distance, $d_{b l}{ }^{a}$ & $3,5,7,9$ \\
Number of blobs, $N_{b l}$ & $1-75$ \\
\hline
\end{tabular}

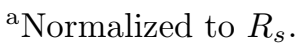


TABLE 2

Summary of THE RESUlts FOR MOdELS WITH A NUMBER OF BLOBS ${ }^{a}$

\begin{tabular}{cccccc}
\hline \hline$N_{b l}$ & $\begin{array}{c}\Delta I \\
(\%)\end{array}$ & $\begin{array}{c}\sigma_{\Delta I} \\
(\%)\end{array}$ & $\begin{array}{c}\bar{P} \\
(\%)\end{array}$ & $\begin{array}{c}\sigma_{P} \\
(\%)\end{array}$ & $\begin{array}{c}\text { Number of } \\
\text { Simulations }\end{array}$ \\
\hline 1 & 16.5 & 0.4 & 0.17 & 0.09 & 10 \\
3 & 18.3 & 4.4 & 0.28 & 0.13 & 10 \\
10 & 26.0 & 4.5 & 0.39 & 0.18 & 10 \\
20 & 28.2 & 2.8 & 0.49 & 0.24 & 8 \\
30 & 27.3 & 3.0 & 0.44 & 0.23 & 3 \\
40 & 26.6 & 1.7 & 0.41 & 0.17 & 3 \\
50 & 26.4 & 3.4 & 0.42 & 0.16 & 3 \\
60 & 22.1 & 2.0 & 0.39 & 0.14 & 3 \\
70 & 22.3 & 2.0 & 0.40 & 0.14 & 4 \\
75 & 18.1 & 1.7 & 0.39 & 0.14 & 4 \\
\hline
\end{tabular}

aThe model parameters are: $\tau_{\text {env }}=0.01$; $\tau_{b l}=5.0 ; R_{b l}=0.5 ; d_{b l}=3.0$. 
TABLE 3

VARIATION IN FLUX CAUSED BY SCATTERING AND EXTINCTION IN AN INHOMOGENEOUS WR ENVELOPE

\begin{tabular}{lllllcc}
\hline \hline$N_{b l}$ & $\tau_{\text {env }}$ & $\tau_{b l}$ & $R_{b l}$ & $d_{b l}$ & $\begin{array}{c}F_{\text {scat }} \\
\%\end{array}$ & $\begin{array}{c}\Delta I \\
\%\end{array}$ \\
\hline 1 & 0.01 & 10. & 1. & 3. & 7.1 & 76 \\
1 & 0.40 & 10. & 1. & 3. & 4.6 & 47 \\
1 & 1.00 & 10. & 1. & 3. & 2.7 & 27 \\
& & & & & & \\
1 & 0.01 & 5. & 0.5 & 3. & 1.9 & 16.5 \\
3 & 0.01 & 5. & 0.5 & 3. & 3.4 & 18.3 \\
10 & 0.01 & 5. & 0.5 & 3. & 8.5 & 26.0 \\
20 & 0.01 & 5. & 0.5 & 3. & 15.7 & 28.2 \\
30 & 0.01 & 5. & 0.5 & 3. & 21.9 & 27.3 \\
40 & 0.01 & 5. & 0.5 & 3. & 24.4 & 26.6 \\
50 & 0.01 & 5. & 0.5 & 3. & 26.3 & 26.4 \\
60 & 0.01 & 5. & 0.5 & 3. & 25.3 & 22.1 \\
70 & 0.01 & 5. & 0.5 & 3. & 23.3 & 22.3 \\
75 & 0.01 & 5. & 0.5 & 3. & 25.4 & 18.1 \\
& & & & & & \\
\hline
\end{tabular}


TABLE 4

Best MOdELS OF INHOMOGENEOUS WR WINDS ${ }^{\mathrm{a}}$

\begin{tabular}{cccccccccc}
\hline \hline$\tau_{\text {env }}$ & $d_{b l}$ & $R_{b l}$ & $\tau_{b l}$ & $\begin{array}{c}\text { Density } \\
\text { Law }\end{array}$ & $\begin{array}{c}\Delta I \\
(\%)\end{array}$ & $\begin{array}{c}\Delta P \\
(\%)\end{array}$ & $\begin{array}{c}\sigma_{\Delta P} \\
(\%)\end{array}$ & $\Delta I / \Delta P$ & $M_{b l}{ }^{\mathrm{b}}$ \\
\hline 0.10 & 3.0 & 0.5 & 2.0 & Unif. & 11.3 & 0.266 & 0.047 & 42 & 0.023 \\
0.20 & 3.0 & 0.5 & 2.0 & Unif. & 10.0 & 0.249 & 0.066 & 40 & 0.011 \\
0.40 & 5.0 & 1.0 & 2.0 & Unif. & 28.8 & 0.335 & 0.071 & 86 & 0.023 \\
0.40 & 3.0 & 1.0 & 1.0 & Unif. & 20.4 & 0.446 & 0.079 & 46 & 0.011 \\
0.50 & 3.0 & 1.0 & 1.0 & $\beta=0.5$ & 9.7 & 0.36 & 0.11 & 27 & 0.016 \\
1.00 & 3.0 & 1.0 & 5.0 & Unif. & 24.8 & 0.514 & 0.062 & 48 & 0.023 \\
1.00 & 3.0 & 1.0 & 2.0 & $\beta=0.5$ & 12.5 & 0.47 & 0.15 & 27 & 0.016 \\
1.00 & 5.0 & 1.0 & 10.0 & Unif. & 23.5 & 0.364 & 0.070 & 65 & 0.045 \\
2.0 & 3.0 & 1.0 & 5.0 & Unif. & 10.6 & 0.548 & 0.057 & 19 & 0.011 \\
\hline
\end{tabular}

${ }^{a}$ All models listed here are for one blob $\left(N_{b l}=1\right)$.

${ }^{\mathrm{b}}$ Mass relative to the total mass of the envelope. 


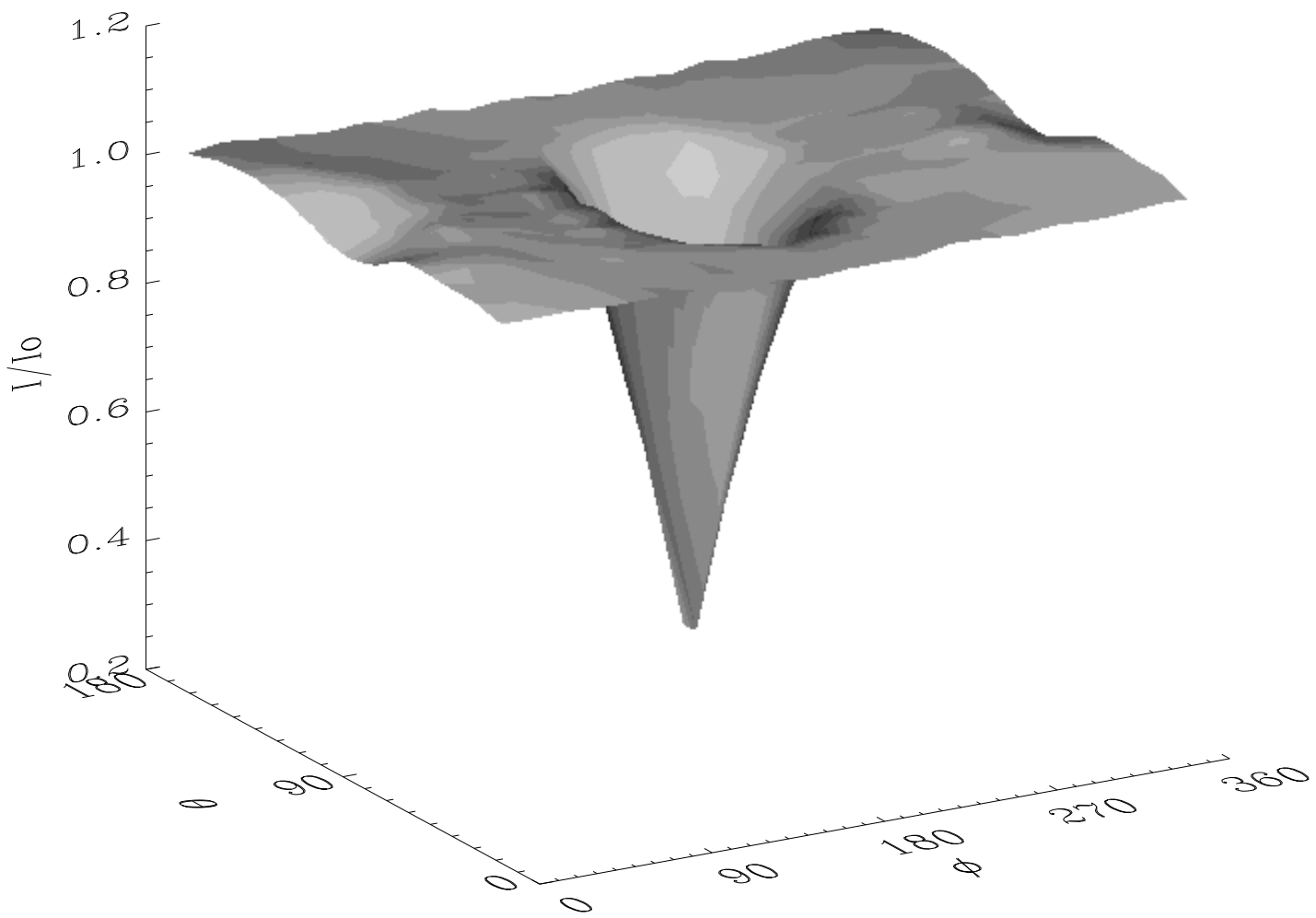




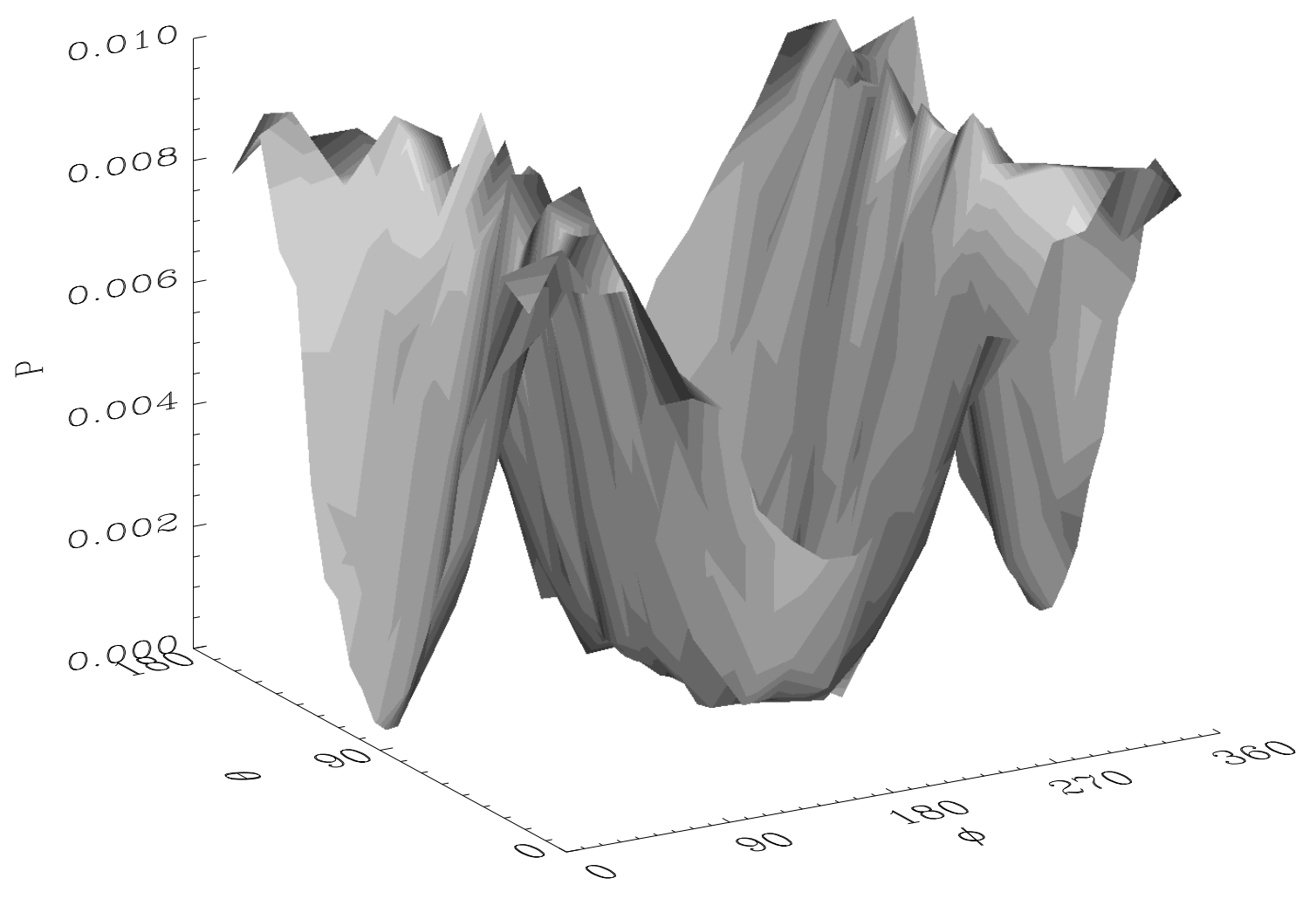




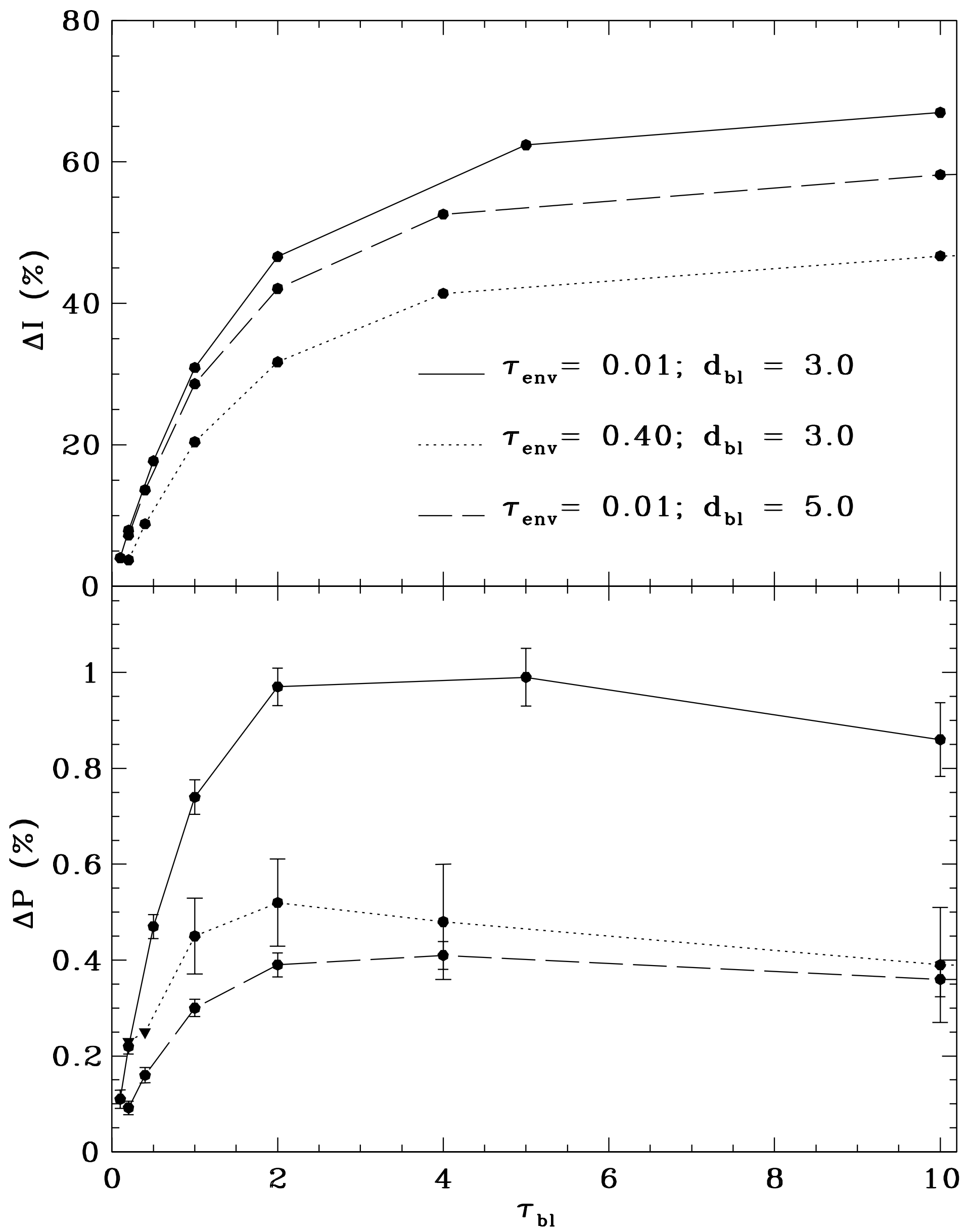




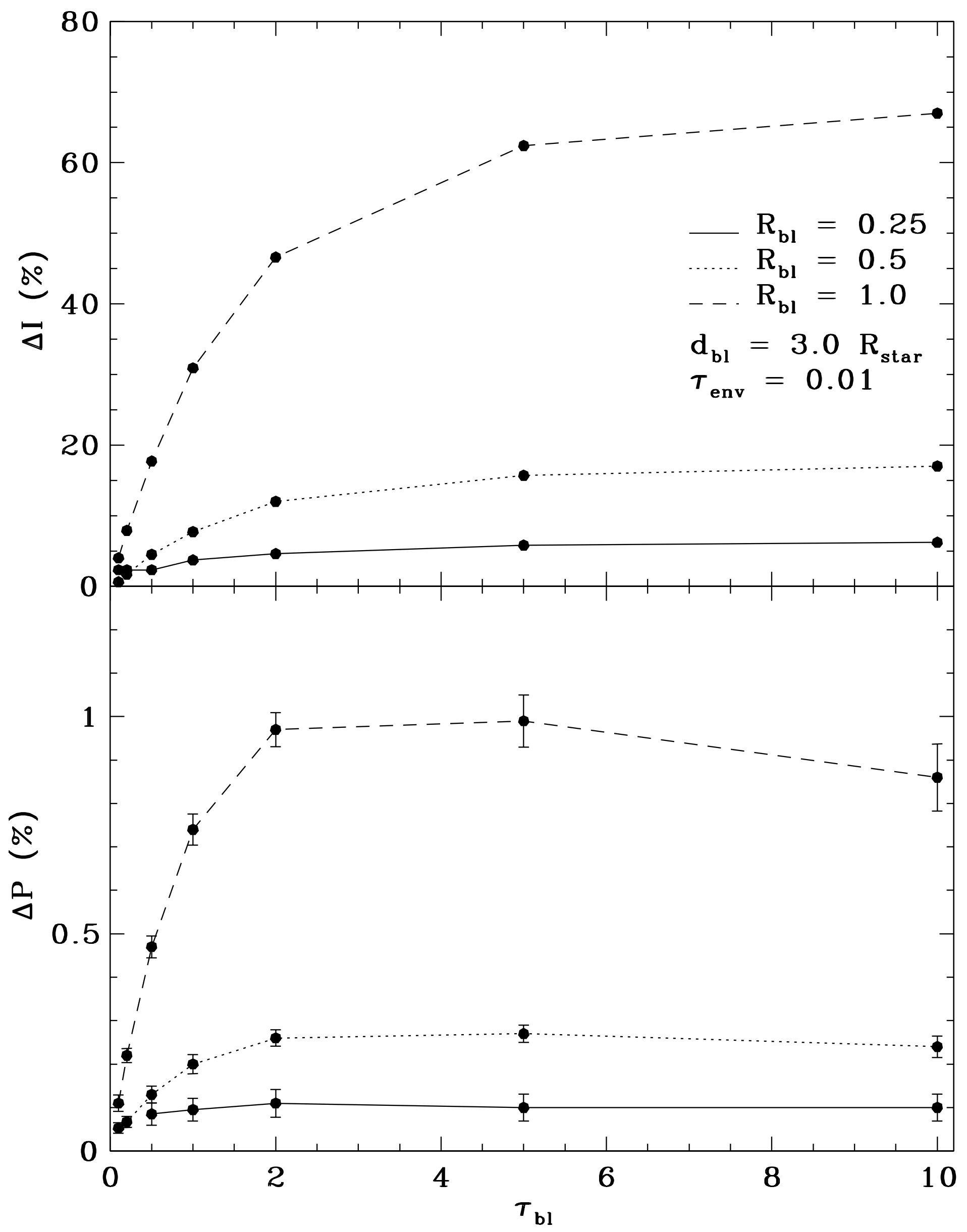




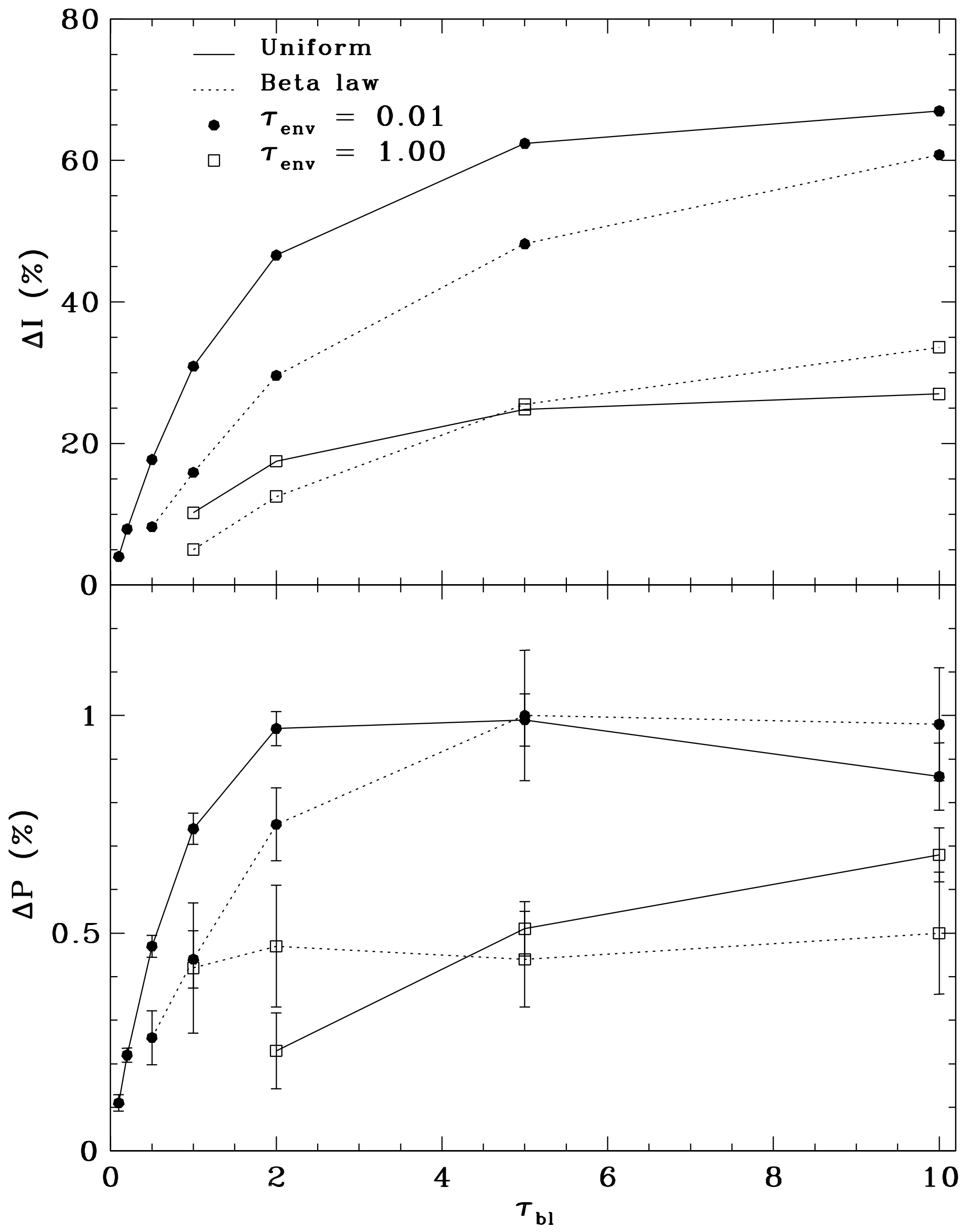




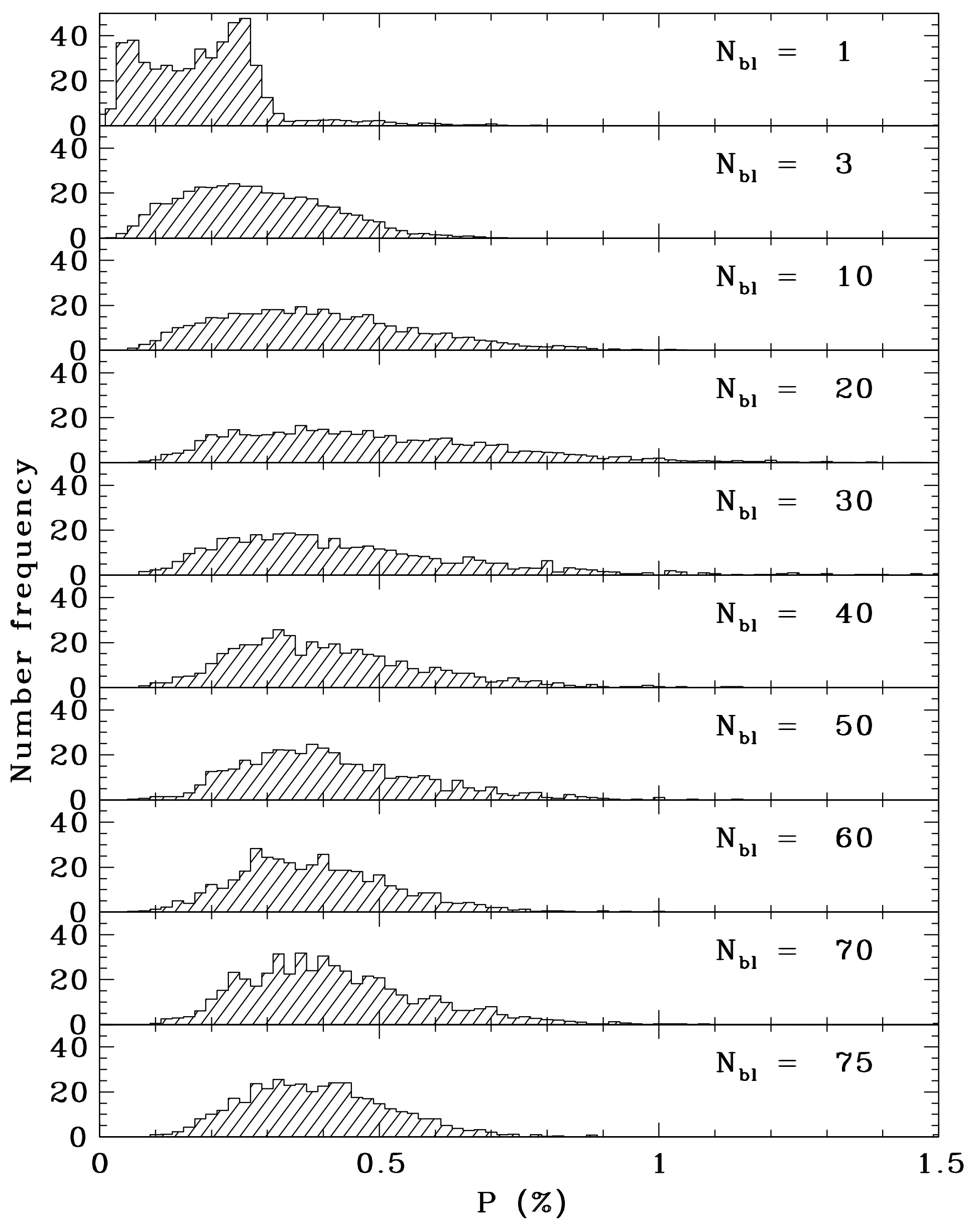

